\title{
COP9 signalosome subunit 5 regulates cancer metastasis by deubiquitinating SNAIL
}

\author{
Kensuke Watanabe ${ }^{1}$, Satoru Yokoyama ${ }^{1}$, Naoki Kaneto ${ }^{1}$, Takashi Hori ${ }^{2}$, Yusuke \\ Iwakami ${ }^{1}$, Shinichiro Kato ${ }^{1}$, Yoshihiro Hayakawa ${ }^{1}$, Hiroaki Sakurai ${ }^{1,3}$, Junya \\ Fukuoka ${ }^{4}$ and Ikuo Saiki ${ }^{1}$ \\ ${ }^{1}$ Division of Pathogenic Biochemistry, Institute of Natural Medicine, University of Toyama, Toyama 930-0194, Japan \\ ${ }^{2}$ Department of Diagnostic Pathology, Toyama University Hospital, Toyama 930-0194, Japan \\ ${ }^{3}$ Department of Cancer Cell Biology, Graduate School of Medicine and Pharmaceutical Sciences, University of Toyama, \\ Toyama 930-0194, Japan \\ ${ }^{4}$ Department of Pathology, Nagasaki University Graduate School of Biomedical Sciences, Nagasaki 852-8501, Japan \\ Correspondence to: Satoru Yokoyama, email: yokoyama@inm.u-toyama.ac.jp \\ Keywords: SNAIL; metastasis; COPS5; deubiquitination; lung cancer
}

Received: April 07, $2017 \quad$ Accepted: March 21, $2018 \quad$ Published: April 17, 2018

Copyright: Watanabe et al. This is an open-access article distributed under the terms of the Creative Commons Attribution License 3.0 (CC BY 3.0), which permits unrestricted use, distribution, and reproduction in any medium, provided the original author and source are credited.

\section{ABSTRACT}

Cancer metastasis is a major cause of mortality in cancer patients. The transcription factor SNAIL plays an important role in cancer metastasis and progression, and its expression is tightly regulated by the ubiquitin-proteasome system through the balance between ubiquitin ligases and deubiquitinating enzymes. While several ubiquitin ligases of SNAIL have been identified, it is not yet clear regarding deubiquitinating enzyme. In this study, we identified COP9 signalosome subunit 5 (COPS5) as a deubiquitinating enzyme of SNAIL by using siRNA library screening. COPS5 downregulation significantly reduced the expression of SNAIL and impaired the metastatic potential of lung cancer cells both in vitro and in vivo. Importantly, we demonstrated that COPS5 binds to SNAIL and stabilizes its expression by deubiquitination. Furthermore, we observed the positive correlation between COPS5 and SNAIL expression in the clinical tissue samples of lung adenocarcinomas by using tissue microarray analysis. These findings provide strong evidence that COPS5 can be a new therapeutic target for cancer metastasis as a deubiquitinating enzyme of SNAIL.

\section{INTRODUCTION}

The critical characteristics of cancers are the ability to invade surrounding tissues and metastasize to distal tissues, which is the major cause of mortality in cancer patients [1]. Cancer metastasis consists of several steps: intravasation, attachment to a vessel, extravasation, angiogenesis, and growth in distal tissues [2]. The migration ability or invasiveness of cancer cells plays a critical role during metastasis.

SNAIL is a transcription factor that regulates the migration ability in some cancer types, including lung and pancreas [3]. Recent studies suggest that SNAIL has a much broader impact on cancer progression such as the induction of epithelial-to-mesenchymal transition [3], an enhancement of recruitment of macrophages [4], an induction of tumor-initiating properties [5], a suppression of host immune surveillance [6], drug resistance [7], and cancer metabolism [8]. Given that SNAIL has multiple functions in cancer progression, it is important to understand the regulatory mechanisms for SNAIL in cancers, resulting in the acquisition of a new therapeutic strategy for cancer progression by targeting SNAIL.

SNAIL has been transcriptionally regulated by some pathways, including transforming growth factor- $\beta$, $\mathrm{NOTCH}$, and WNT pathways, reactive oxygen species, and hypoxic stress [9]. In addition to transcriptional regulation, SNAIL has been regulated post-translationally through 
the ubiquitin-proteasome system. Protein ubiquitination is an essential modification and is tightly regulated by the balance between ubiquitination and deubiquitination. In the case of SNAIL, at least four E3 ubiquitin ligases, FBXO11 [10], FBXW1 [11], FBXL5 [12], and FBXL14 $[13,14]$, have been identified as the responsible enzymes for SNAIL ubiquitination and degradation. In contrast, few deubiquitinating enzymes (DUBs) for SNAIL has been identified [15], though approximately 100 putative DUBs are known in human [16]. A protein ubiquitination has been classified into three groups, K48-linked, K63-linked, and M1-linked polyubiquitination. K48-linked and K63linked polyubiquitination have been mainly implicated in proteasomal degradation or endosomal sorting/lysosomal targeting, respectively. Even in DUBs, their substrates have been shown to have specificities to K48-, K63-, or M1-linked polyubiquitination.

In this study, we identify COP9 signalosome subunit 5 (COPS5), one of the DUBs, as a deubiquitinating enzyme of SNAIL by HA-based siRNA screening. COPS5 knock-down shows a significant inhibition of invasiveness in vitro and also lung metastasis in vivo. By functional studies, we determine the COPS5-mediated stabilization of SNAIL through its deubiquitination. Furthermore, the positive correlation between COPS5 and SNAIL expression is observed in the clinical tissue samples of lung adenocarcinomas using tissue microarray analysis. Collectively, COPS5 could be a good molecular target for cancer metastasis through SNAIL degradation.

\section{RESULTS}

\section{Deubiquitinating enzymes regulate SNAIL expression and invasion in cancer cells}

SNAIL plays a critical role in migration ability and invasiveness in human lung adenocarcinoma A549 cells and pancreatic ductal adenocarcinoma Panc-1 cells in our previous reports [17]. In addition, the expression of SNAIL has also been known to be regulated by an ubiquitinproteasome system (UPS) [13]. Indeed, we observed that the reduction or the accumulation of SNAIL protein in human lung adenocarcinoma A549 cells and pancreatic ductal adenocarcinoma Panc-1 cells occurred after treatment with a protein synthesis inhibitor, cycloheximide (CHX) (Figure 1A), or treatment with a proteasome inhibitor, MG132 (Figure 1B), respectively. These results confirmed that SNAIL expression is regulated by UPS and the degradation machinery of SNAIL can be intact in those cancer cell lines.

We then focused on deubiquitinating enzymes (DUBs), which reverses protein ubiquitination and finally stabilizes target proteins. To test whether DUBs are involved in the stability of SNAIL protein in cancer cells, we used a pan-DUB inhibitor, PR-619. While total ubiquitinated protein were accumulated after PR-619 treatment, SNAIL protein was significantly reduced in both A549 and Panc-1 cells (Figure 1C). Along with the reduction of SNAIL expression, the invasiveness of both A549 and Panc-1 cells were largely impaired by PR619 treatment (Figure 1D). The effects of PR-619 on the invasiveness were stronger than that on the cell growth (Supplementary Figure 1). These results indicate that DUBs regulate SNAIL degradation and cancer cell invasiveness.

\section{Identification of deubiquitinating enzymes targeting SNAIL}

To identify potential DUBs responsible for SNAIL protein stability in an unbiased manner, we established HA/SNAIL-overexpressing HeLa cells (HeLa-HA/ SNAIL) using pIRES2-HA/SNAIL, in which HA/SNAIL and EGFP are transcribed in a single mRNA and separately translated through bicistronic expression. Consequently, we could determine the post-translational regulation of SNAIL by comparing HA/SNAIL expression with EGFP expression. By knocking-down of individual 97 human DUBs by pooled siRNA (four siRNAs per each gene) in HeLa-HA/SNAIL, we found 20 and 15 genes from first screening and second screening, respectively. From these two independent experiments, 5 siRNAs (COPS5, JOSD1, OTUB1, OTUD7A, and OTUD7B) were commonly identified, all of which suppressed both HA/SNAIL and endogenous SNAIL expression compared with control knocked-down cells (siCNTL) (Figure 2A and 2B). We further evaluated the endogenous SNAIL protein in A549 cells by using two individual siRNAs for each gene. Amongst them, COPS5 knockdown could strongly suppress endogenous SNAIL expression (Figure 2C); therefore, we focused on COPS5 in further experiments.

\section{COPS5 regulates the invasiveness and metastasis of lung cancer cells.}

To confirm the functional significance of SNAIL suppression by COPS5 knock-down, we next examine the migration ability and invasiveness using A549 cells and Panc-1 cells. Strikingly, the knockdown of COPS5 significantly decreased both the migratory ability and invasiveness of both A549 and Panc-1 cell lines (Figure 3A and $3 \mathrm{~B}$ ), in concert with the reduction of SNAIL and its downstream target protein, Vimentin (Figure 3C) [18]. Because both A549 and Panc-1 are KRAS mutant cancer cell lines, we next checked the effects of COPS5 knockdown in H1650 or H2228 lung adenocarcinoma cell lines, which has an EGFR mutation or EML4-ALK fusion, respectively. As shown in Figure 3D, their invasiveness were significantly suppressed by knocking-down of COPS5 in both H1650 and H2228 in concert with SNAIL reduction, suggesting the important role of COPS5 in the invasiveness of lung cancers independent of their specific oncogenic mutations. 
Next, we tested the functional impact of COPS5 on lung cancer metastasis in vivo by using A549 cells expressing the firefly luciferase gene (A549/Luc2) [17]. Consistent with the in vitro results, the knockdown of COPS5 in A549/Luc2 cells significantly suppressed the formation of lung metastasis compared to the control knockdown in A549/Luc2 cells (Figure 3E). We also detected SNAIL reduction after the knockdown of COPS5 in A549/Luc2 cells (Supplementary Figure 2). These results clearly indicate that COPS5 is involved in the lung metastatic ability of cancer cells even in the physiological condition.

\section{COPS5 directly regulates SNAIL stability through deubiquitination.}

To directly address whether COPS5 stabilizes SNAIL protein, A549 cells overexpressing Flag-tagged COPS5 (Flag/COPS5) were treated with CHX, an inhibitor of protein synthesis. As shown in Figure 4A, the half-life of SNAIL protein upon CHX treatment was prolonged by the overexpression of Flag/COPS5 compared with the vector control. To further investigate whether COPS5 functions as a DUB for regulating SNAIL expression, we examined the effects of COPS5 overexpression on SNAIL ubiquitination in A549 cells by immunoprecipitation assay. While the ubiquitination of HA/SNAIL was observed, it was significantly decreased by co-expressing Flag/ COPS5 in A549 cells (Figure 4B). Furthermore, coimmunoprecipitation between Flag/COPS5 and HA/ SNAIL in A549 cells (Figure 4C) and co-localization of COPS5 with SNAIL in immunofluorescence staining (Figure 4D and Supplementary Figure 3) supported the direct association of COPS5 and SNAIL. Collectively, these results indicate that COPS5 can be a bona fide deubiquitinating enzyme of SNAIL protein.

To investigate the significance of SNAIL deubiquitination by COPS5 in cancer metastasis, we introduced the mutant forms of SNAIL protein (K98R/ K137R/K146R, referred to as SNAIL-3KR) in A549 cells,
A

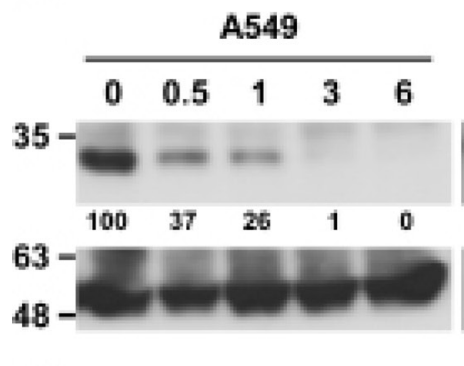

C

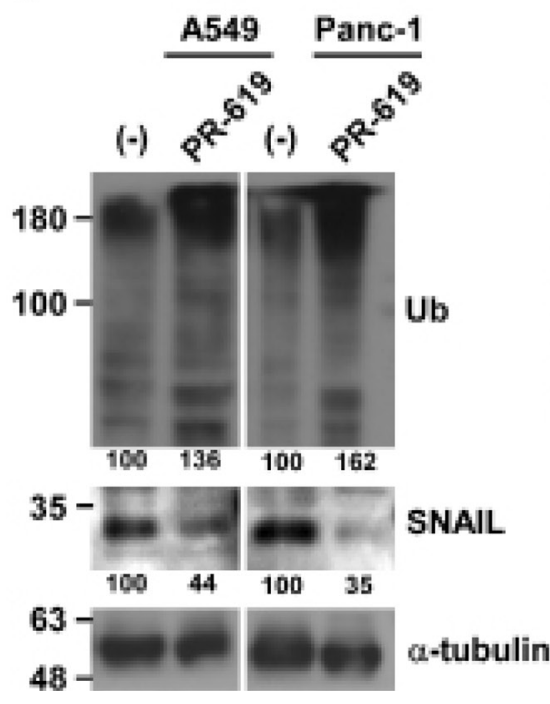

B

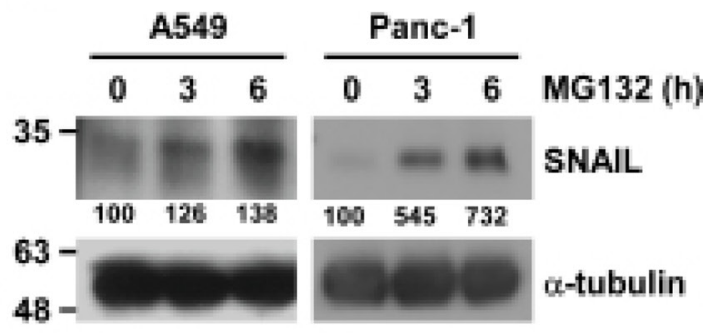

Figure 1: Inhibition of deubiquitinating enzymes suppressed cancer cell invasion. (A) A549 or Panc-1 cells were treated with $50 \mu \mathrm{g} / \mathrm{ml}$ cycloheximide (CHX) for the indicated times and subjected to Western blotting. The band intensities were measured by ImageJ, normalized to that at $0 \mathrm{~h}$ of each cells, and shown below each panel. (B) A549 or Panc-1 cells were treated with $10 \mu \mathrm{M}$ MG132 for the indicated times and subjected to Western blotting. Other conditions were similar to Figure 1A. (C, D) A549 or Panc-1 cells were treated with $75 \mu \mathrm{M}$ PR-619 or DMSO for $3 \mathrm{~h}$ and subjected to Matrigel invasion assay (C) or Western blotting (D). Invaded cells were counted and normalized with the vehicle control $(-)$. Data are represented as the mean \pm S.D. of at least three independent experiments. ${ }^{*} P<0.01$ vs DMSO-treated cells by two-tailed student's $t$ test. 
which lacks three ubiquitination sites [13] and therefore increased protein stability (data not shown). As shown in Figure 4E, SNAIL-3KR partially, but statistically significantly, rescued the impaired invasion of A549 cells by COPS5 knock-down. These results indicate the role of endogenous COPS5 in SNAIL-dependent cancer cell invasion through the deubiquitinating activity of COPS5.

Finally, to explore the clinical relevance of the present findings, we examined the expression of COPS5 and SNAIL in the tissue microarray containing 160 lung adenocarcinomas (Figure 5 and Supplementary Figure 4). In alignment with our presented findings in the cell lines, the higher levels of COPS5 expression were correlated with SNAIL expression in the lung adenocarcinoma tissue samples as determined by the immunohistochemistry scoring. Collectively, these results strongly support the association between COPS5 and SNAIL expression in the clinical cancer samples likely through the deubiquitinating activity of COPS5.

\section{DISCUSSION}

The significance of SNAIL functions is not only known in cancer metastasis but also in epithelial-tomesenchymal transition (EMT) [3], cancer stem cell properties [5], immune evasion [6], and cancer metabolism [8]. The SNAIL protein stability, cellular localization, and activity are known to be regulated by post-translational modifications such as phosphorylation, acetylation, and ubiquitination. Although there are several reports regarding the ubiquitin ligases of SNAIL, little is known about the deubiquitinating enzyme of SNAIL, which reverses its ubiquitination and therefore stabilizes the protein expression of SNAIL. Here, we identified COPS5 as a deubiquitinating enzyme (DUB) of SNAIL to regulate the metastatic ability of lung cancer cells. The positive correlation between SNAIL and COPS5 expression was also relevant in the tissue microarray analysis of human lung adenocarcinoma panel. Importantly, COPS5 knockdown did induce epithelial-like phenotype in A549 cells, as seen in the reduction of vimentin expression (Figure 3C) and the morphological changes from spindlelike shape to cobblestone-like shape (data not shown). These results clearly show the importance of COPS5 as a deubiquitinating enzyme of SNAIL in the metastatic progression of cancer cells.

COPS5, also known as CSN5 or JAB1, is one of the subunits of COP9 signalosome, which is a highly conserved protein complex between the species [19]. COPS5 is a multifunctional protein that plays

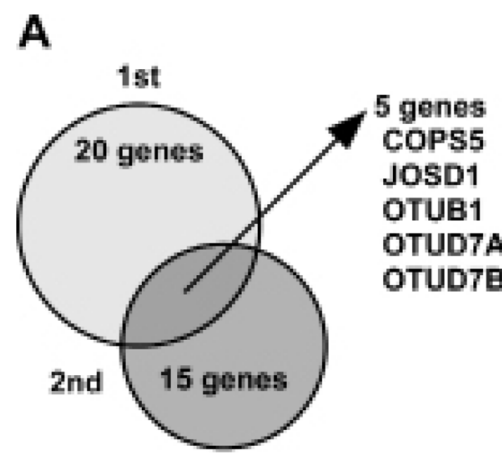

B
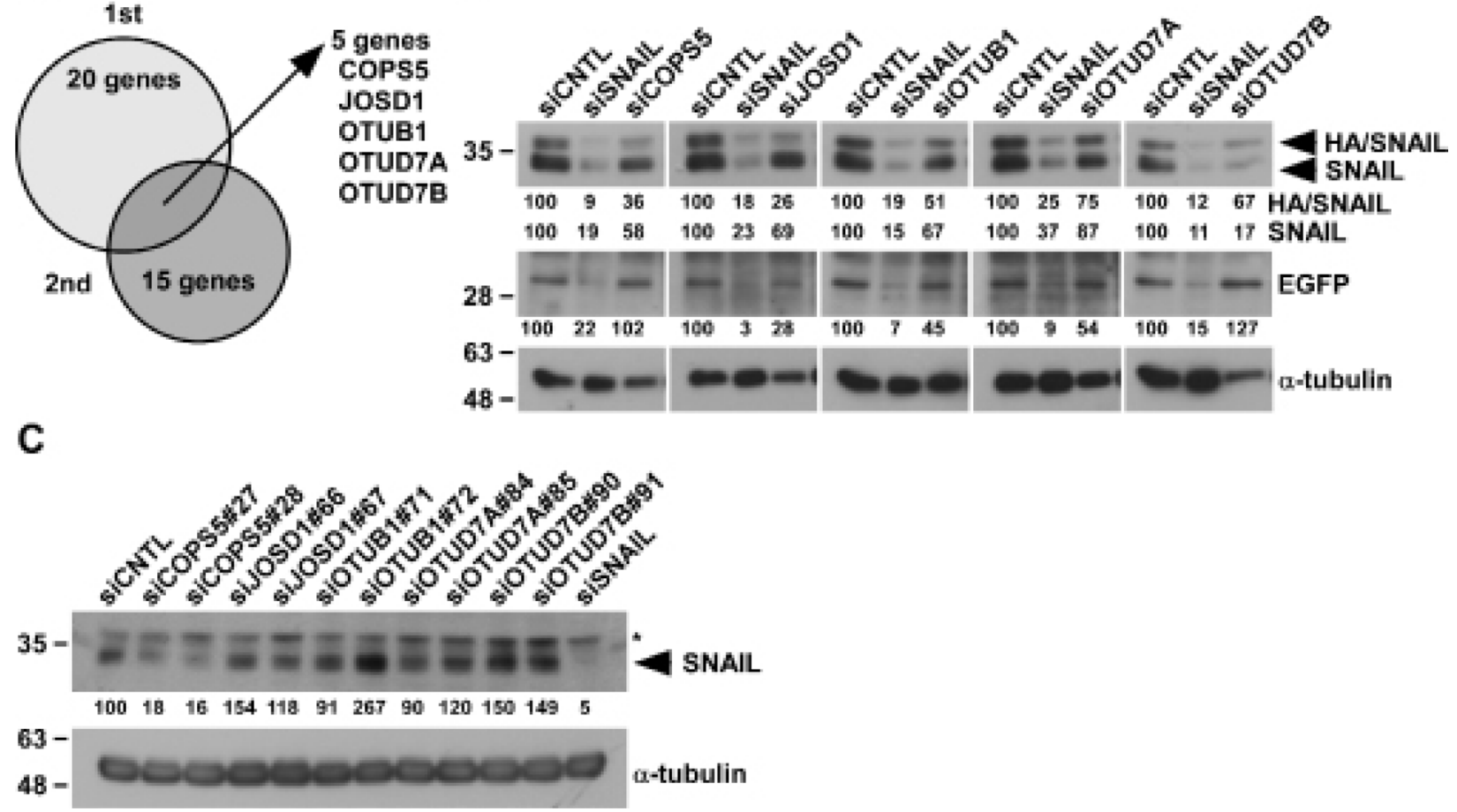

Figure 2: Identification of deubiquitinating enzymes targeting SNAIL. (A) $12.5 \mathrm{nM}$ siRNAs against each DUB were transfected to HeLa-HA/SNAIL for $96 \mathrm{~h}$ and whole cell lysates were subjected to Western blotting. Biological duplicate experiments were performed and 5 candidates were identified as common ones in two experiments. (B) The pictures are the results of Western blotting in HeLa-HA/ SNAIL cells after knocking-down of SNAIL or 5 candidates, respectively. The band intensities were measured by ImageJ, normalized to that of siCNTL-transfected cells, and shown below each panel. (C) A549 cells transfected with the indicated siRNA for $96 \mathrm{~h}$ were subjected to Western blotting. "shows non-specific band using anti-SNAIL antibody. Other conditions were similar to Figure $2 \mathrm{~B}$. 
an important role in the regulation of chromosome instability, DNA damage, and cell cycle [20] targeting K48 and K63-linked polyubiquitination [21]. Dysregulation of COPS5 activity has been shown to contribute to oncogenesis through its function in cell proliferation [22], cell cycle progression [23], and cell chemo-/radio-resistance [21, 24], that are mediated by the various target molecules such as MDM2, p27, HIF$1 \alpha$, and AP-1 [25]. Recently, the relevance of COPS5 amplification and overexpression in the tamoxifenresistance of ER $\alpha$-positive breast cancer was also reported [21]. Although little is known regarding the involvement of COPS5 in cancer metastasis, the role of COPS 5 in the invasion of human colorectal cancer cell lines was previously studied [26]. In addition to its direct contribution in cancer cell invasion, COPS5 is known to regulate TGF- $\beta$ signaling by binding to Smad7 [27], which is the important signaling pathway for both
A

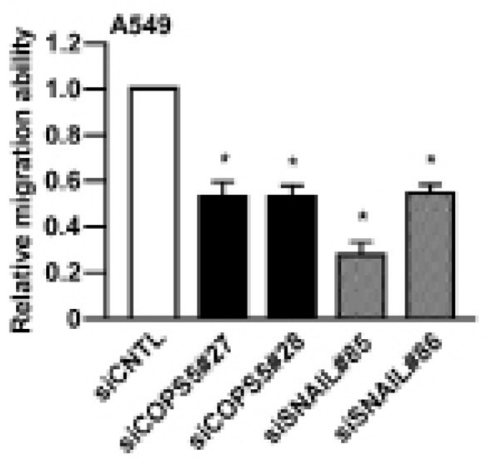

B

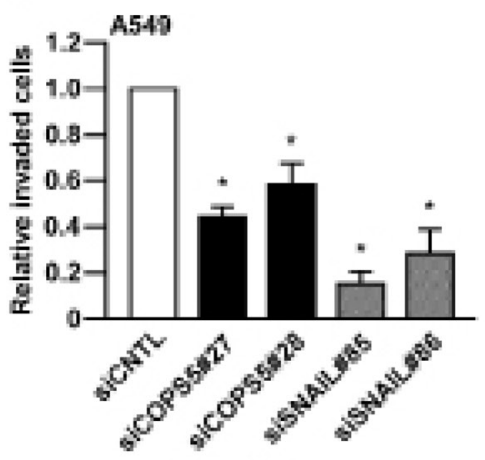

D

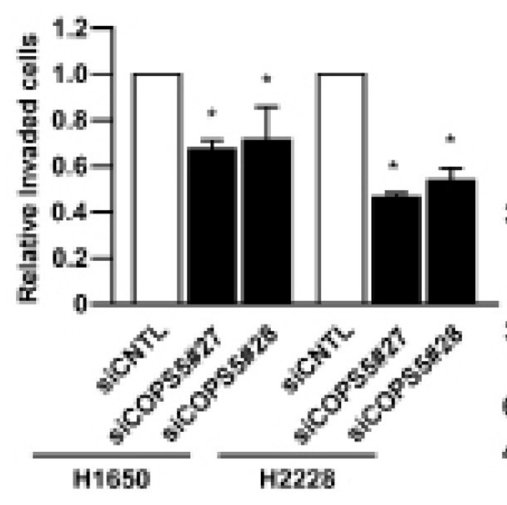

C
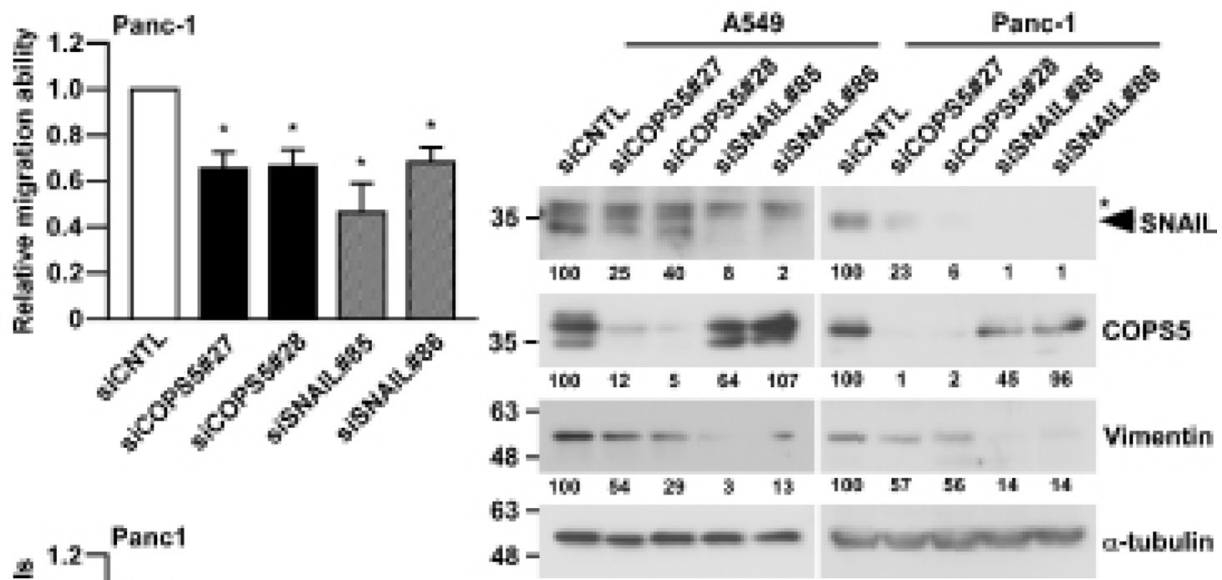

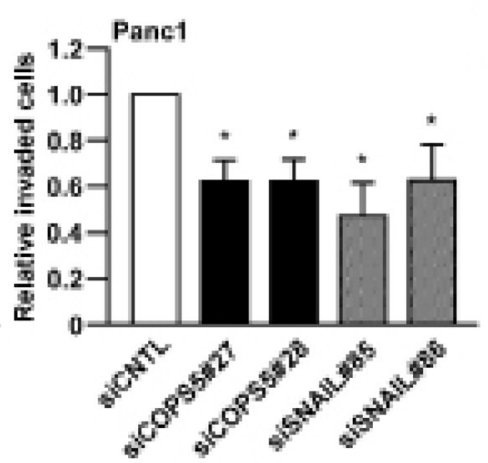

E
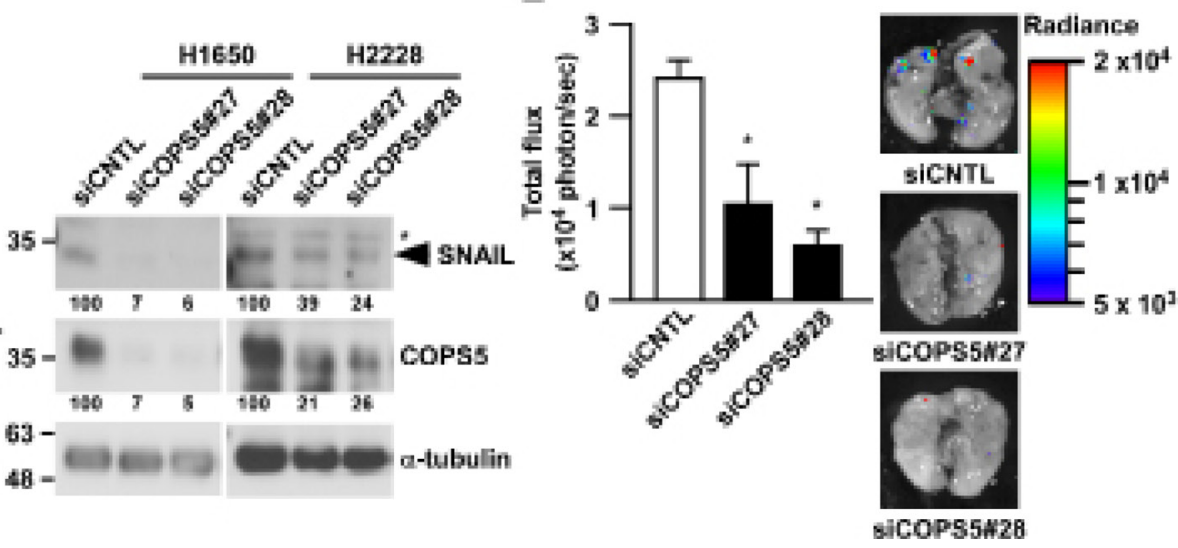

Figure 3: COPS5 regulates the invasiveness and metastasis of lung cancer cells. (A-C) A549 and Panc-1 cells transfected with the indicated siRNA for $96 \mathrm{~h}$ were subjected to Wound healing assay (A), Matrigel-invasion assay (B), and Western blotting (C). The band intensities were measured by ImageJ, normalized to that of siCNTL-transfected cells, and shown below each panel. "shows nonspecific band using anti-SNAIL antibody. (D) Two lung adenocarcinoma cells (H1650 and H2228) transfected with the indicated siRNA for $96 \mathrm{~h}$ were subjected to Matrigel-invasion assay (left panel) or Western blotting (right panel). (E) A549/Luc2 cells transfected with the indicated siRNA for $96 \mathrm{~h}$ were subjected to In vivo invasion assay $(n=3$, each group). Data are represented as the mean \pm S.D. of at least three independent experiments. ${ }^{*} P<0.01$ vs siCNTL-transfected cells by one-way ANOVA followed by the Bonferroni post-hoc test. 
A

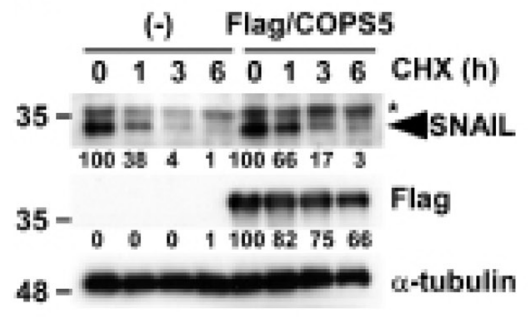

B

$(-)(+)(-)$ Flag/COPS5

$(\mathrm{kDa})(+)(+)(-)$ HAJSNAIL

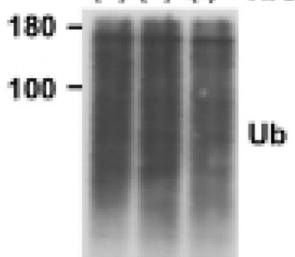

10010469

$180-$

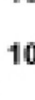

- Flag/Cops5

$100-$

$-\pi$

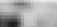

$100 \div 2$

HA

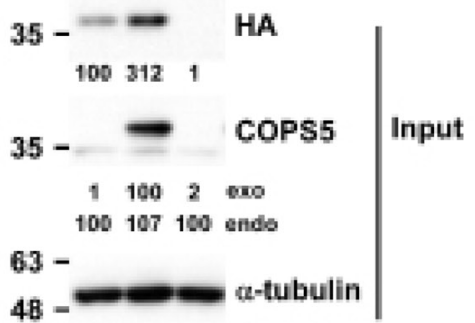

D

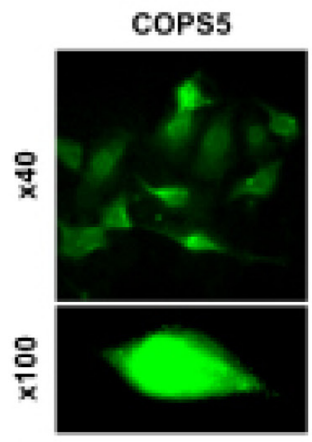

SNAIL
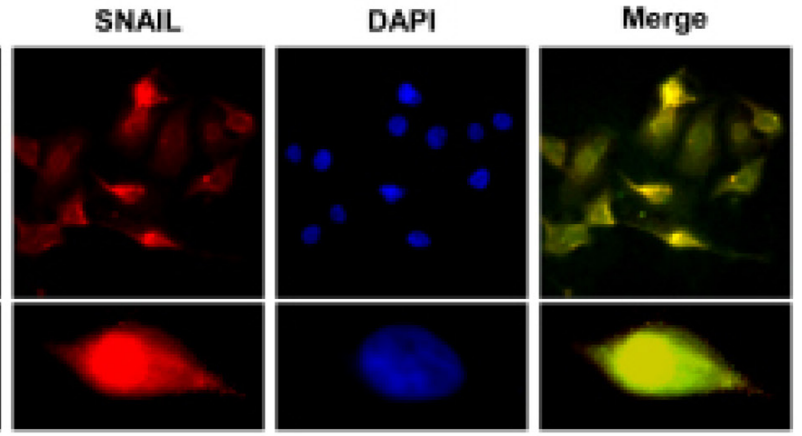

E

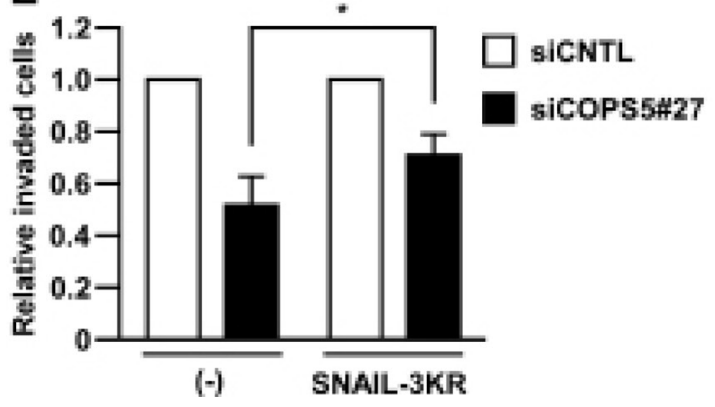

Figure 4: COPS5 directly regulates SNAIL stability through deubiquitination. (A) A549 cells transfected with Flag-tagged COPS5 (Flag/COPS5) or empty vector (-) were treated with cycloheximide (CHX) for the indicated time and subjected to Western blotting (upper panels). "shows non-specific band using anti-SNAIL antibody. The band intensities in biological triplicated experiments were measured by ImageJ and normalized to that at $0 \mathrm{~h}$ of each transfected cells (lower panel). Data are represented as the mean \pm S.D. of three independent experiments. ${ }^{* *} P<0.01$ or ${ }^{*} P<0.05$ vs non-transfected cells by two-way ANOVA followed by the Bonferroni post-hoc test. (B) A549 cells were transfected with either Flag/COPS5 or HA/SNAIL for $48 \mathrm{~h}$. After immunoprecipitation of the ubiquitinated protein, the cell lysates were subjected to Western blotting. The band intensities were measured by ImageJ and normalized to that of each control. (C) A549 cells were transfected with both Flag/COPS5 and HA/SNAIL for $48 \mathrm{~h}$. After immunoprecipitation using anti-Flag antibody (left panel) or anti-HA antibody (right panel), the cell lysates were subjected to Western blotting. Other conditions were similar to Figure 4B. (D) A549 cells were treated by MG132 for $3 \mathrm{~h}$ and stained by SNAIL (red), COPS5 (green), and DAPI (blue), respectively. The fluorescent images are shown as pictures under a microscope at $\times 40$ (upper panels) or at $\times 100$ (lower panels). (E) A549 cells transfected with each siRNA ( $96 \mathrm{~h}$ ) and HA/SNAIL-3KR ( $48 \mathrm{~h}$ ) were subjected to Matrigel Invasion assay. Data are represented as the mean \pm S.D. of at least three independent experiments. ${ }^{~} P<0.01$ vs siCNTL-transfected cells by two-way ANOVA followed by the Bonferroni post-hoc test. 
metastasis and EMT. The overexpression of COPS5 promoted the degradation Smad7, and is therefore considered to increase Smad2 phosphorylation to enhance TGF- $\beta$-induced transcriptional activity. Although the potential involvement of Smad7 in COPS5-dependent metastasis regulation still needs to be determined, we observed the sustained SNAIL mRNA expression (data not shown) even after COPS5 knock-down, supporting the post-translational SNAIL regulation by COPS5. In addition, COPS5 is also known as a common component of various smaller complexes containing some other subunits of COP9 signalosome [28] such as CSN2 and CSN6. While CSN2 has been shown to regulate SNAIL stability through inhibiting its phosphorylation by GSK$3 \beta$ [29], CSN6 has been shown to be overexpressed in colorectal cancer and involved in its development [30]. COPS5 has also reported to suppress the SNAIL ubiquitination by inhibiting the binding of SNAIL with $\beta$-Trcp, an E3 ligase [29]. As we observed that the ectopic expression of non-ubiquitinated mutant SNAIL (SNAIL-3KR) enhanced the impaired invasion of COPS5 knock-down A549 cells (Figure 4E), the ubiquitination of SNAIL at least in part affects SNAIL function and the metastatic potential of cancer cells. Moreover, only partial rescue by the mutant SNAIL in our study implies the sustained SNAIL ubiquitination and degradation through additional ubiquitination sites such as K85 and K234 [31]. Although the effects of COPS5 knock-down in SNAIL expression was most predominant amongst the candidates in lung adenocarcinomas, the other four DUBs (JOSD1, OTUB1, OTUD7A, and OTUD7B) could potentially regulate SNAIL expression in other cancer such as cervical cancers (Figure 2). Indeed, OTUB1 has been reported to promote metastasis by facilitating
EMT [32]. Nevertheless, COPS5, as well as COP9 signalosome, can be an attractive molecular target for cancer metastasis through the regulation of the SNAIL pathway.

Regarding the positive correlation between COPS5 and SNAIL expression in lung adenocarcinoma tissues (Figure 5), we also found the correlation between high COPS5 expression and poor survival of lung adenocarcinoma patients by analyzing the GEO datasets (GSE13213 and GSE31210) [33, 34] in Prognoscan (Supplementary Figure 4) [35]; therefore, COPS5 could be a prognostic marker for lung cancer patients. Strikingly, it is demonstrated that non-cancerous lung tissues showed significantly lower COPS5 expression than those in lung cancer tissues [36]. Moreover, these clinical data, which do not specify any oncogenic mutation, and our invasion experiments using some lung cancer cells with different mutation including KRAS (A549), EGFR (H1650), and EML4-ALK (H2228) (Figure 3D) imply that the target therapy for COPS5 may be applicable to the lung cancer patients even with different mutation types. Besides lung cancer [37], COPS5 is overexpressed in many other types of cancer, including breast [38], pancreatic [39], and hepatocellular carcinoma [40]. Not only its overexpression, but also COPS5 gene amplification has been recently reported in ER $\alpha$-positive breast cancer, supporting that COPS5 might be an oncogene in some cancers [21]. The important role of SNAIL in cancer progression and metastasis is also relevant to many types of cancer other than lung cancer [3]. Collectively, our present study sheds light on the COPS5 deubiquitinating enzyme to control SNAIL-dependent metastasis and progression of COPS5overexpressing cancers, and further implicates the new therapeutic strategy of cancer by targeting COPS5.
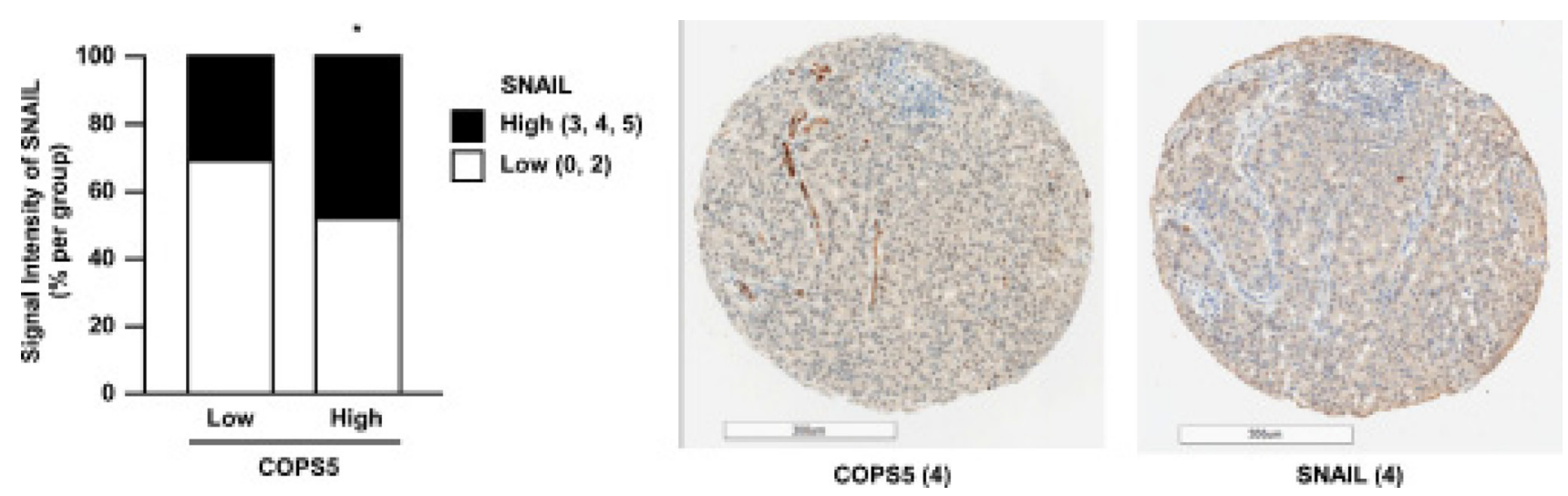

Figure 5: COPS5 expression is positively correlated with SNAIL expression in lung adenocarcinoma tissues. A lung adenocarcinoma tissue microarray, including 200 cores, was adopted for immunohistochemical staining using primary antibodies against SNAIL and COPS5. Nuclear and/or cytoplasmic staining for SNAIL or COPS5 was considered to indicate positivity. Image capture and evaluation were performed in a masked manner by two independent researchers. Among 200 cores containing lung adenocarcinoma tissues, 168 cores were scored by two independent researchers. Finally, 160 cores were classified into same groups by two researchers and used as available cores. The sum of the distribution score (0-2) and the intensity score (0-3) was converted into negative (DS + IS; 0 or 2$)$ and positive (DS + IS; 3-5). The correlation between COPS5 and SNAIL expression was evaluated by Chi-square test among the patient subgroups. ${ }^{*} P<0.05$ was considered statistically significant. Typical staining images of lung cancer tissues are shown. Scale bar, $300 \mu \mathrm{m}$. 


\section{MATERIALS AND METHODS}

\section{Reagents and plasmid preparation}

A protein synthesis inhibitor, cycloheximide, was purchased from Cayman Chemical (Ann Arbor, MI, USA). A proteasome inhibitor (MG132) and a non-selective, reversible inhibitor of deubiquitination (PR-619) were purchased from Merck Millipore (Billerica, MA, USA).

Human SNAIL or COPS5 cDNA was amplified by RT-PCR from cDNA derived from A549 cells and inserted into pIRES2-EGFP (Takara, Shiga, Japan) and pcDNA3.1-HAHA vector (a kind gift from David E. Fisher, MGH, Boston, MA, USA), or p3xFLAG-CMV7.1 vector, respectively. Point mutations of K98R, K137R, and $\mathrm{K} 148 \mathrm{R}$ were introduced by following the protocol of the QuickChange site-directed mutagenesis kit (Stratagene, La Jolla, CA, USA), named pcDNA3.1-HA/SNAIL-3KR.

\section{Cell cultures}

Human cervical cancer cell lines (HeLa), human lung adenocarcinoma cell lines, (A549), and human pancreatic ductal adenocarcinoma cell lines (Panc-1) were obtained from the American Type Culture Collection (ATCC, Rockville, TX, USA). Two human lung adenocarcinoma cell lines (H1650 and H2228) were kindly gifted by Dr. Yano, S. (Kanazawa University, Ishikawa, Japan). All cell lines were maintained in RPMI1640, containing 10\% FBS, $1 \mathrm{mM} \mathrm{L}$-glutamine, and antibiotics (100 units $/ \mathrm{mL}$ penicillin and $100 \mathrm{mg} / \mathrm{mL}$ streptomycin) in a humidified atmosphere of $95 \%$ air and $5 \% \mathrm{CO}_{2}$ at $37^{\circ} \mathrm{C}$.

To establish HeLa cells stably expressing HA/SNAIL, HeLa cells were transfected with pIRES2-HA/SNAIL, selected, and cloned in growth medium containing $1 \mathrm{mg} / \mathrm{mL}$ G418. A549/Luc2 cells were previously established [17].

For siRNA screening, $12.5 \mathrm{nM}$ Dharmacon siGENOME SMARTpool siRNA Library (Human Deubiquitinating Enzymes) and siGENOME Non-targeting siRNA Pool \#2 (Thermo Fisher Scientific, Rockford, IL, USA) were transfected in HA/SNAIL-overexpressing HeLa cells (HeLa-HA/SNAIL) using Lipofectamine RNAiMAX reagent (Thermo Fisher Scientific). For knocking down experiments, Silencer Select Negative Control \#1, or Silencer Select siRNAs against COPS5 (s21627 and s21628), JOSD1 (s19266 and s19267), OTUD7A (s46284 and s46285), and OTUD7B (s32490 and s32491) (Thermo Fisher Scientific) were transfected in A549 and Panc-1 cells using Lipofectamine RNAiMAX reagent. The transfected cells were subjected to a Matrigel invasion assay, Wound healing assay, or Western blotting after $96 \mathrm{~h}$.

For transient transfection, expression vectors were co-transfected into A549 cells using Lipofectamine 2000 reagent (Thermo Fisher Scientific) and the transient transfected cells were subjected to a Matrigel invasion assay or Western blotting after $24 \mathrm{~h}$.
Western blotting and immunoprecipitation assay

Whole cell lysates were prepared as described previously [17]. Primary antibodies used were SNAIL, COPS5, Vimentin from Cell Signaling Technology (Beverly, MA, USA), $\alpha$-tubulin from Santa Cruz Biotechnology (Santa Cruz, CA, USA), anti-FLAG antibody (Sigma-Aldrich, St. Louis, MO, USA), and anti-HA antibody from Roche (Indianapolis, IN, USA). The ubiquitination of SNAIL was assessed by Ubiquitin Enrichment Kit (Thermo Fisher Scientific) according to the manufacturer's instructions. The immunoprecipitation lysates were subjected to Western blotting. The band intensities were measured by ImageJ and normalized to that of each control lane.

\section{Wound healing assay}

Wound healing assay was performed as described previously [41]. Briefly, A549 and Panc- 1 cells $\left(1 \times 10^{5}\right.$ cells/ well) were seeded into a 24-well plate and treated $12.5 \mathrm{nM}$ siRNA transfection. After $96 \mathrm{~h}$ siRNA transfection, confluent cells were scratched using pipette tips. After scratching, the scratched width was measured at $0 \mathrm{~h}$ and $48 \mathrm{~h}$.

\section{Matrigel invasion assay}

Matrigel invasion assay was performed as described previously [17]. Briefly, membrane filters (Whatman, Maidstone, UK) were attached to Transwell chambers (Costar, Cambridge, MA, USA) and the lower surface was pre-coated with $1.25 \mu \mathrm{g}$ fibronectin (Iwaki, Tokyo, Japan) and the upper surface was pre-coated with $5 \mu \mathrm{g}$ Matrigel (BD Biosciences, Franklin Lakes, NJ, USA). Pre-treated cells $\left(3 \times 10^{4}\right.$ cells $/ 100 \mu 1$ in RPMI 1640 Medium with $0.1 \%$ bovine serum albumin) were added to the upper compartment of the chamber. After incubation for $6 \mathrm{~h}$, the invaded cells were stained by hematoxylin and eosin and counted manually under a microscope at 50x magnification.

\section{Experimental lung metastasis experiment}

Experimental lung metastasis experiments were performed as described previously [17, 42]. C.B-17/ lcrHsd-Prkdcscid mice were purchased from Japan SLC (Hamamatsu, Japan). All experiments were approved and performed according to the guidelines of the Care and Use of Laboratory Animals of the University of Toyama. Cells were inoculated intravenously $\left(2 \times 10^{6}\right.$ cells $/ 200$ $\mu \mathrm{L}$ PBS/mouse) into mice and $200 \mu \mathrm{L}$ of luciferin (1.5 $\mathrm{mg} / \mathrm{mL}$ [VivoGlo; Promega, Madison, WI, USA]) was intraperitoneally injected into mice at $24 \mathrm{~h}$ after the tumor inoculation. After $20 \mathrm{~min}$, the lungs were removed to subject bioluminescent assay by using an in vivo imaging system (IVIS Lumina II, Caliper Life Sciences, Hopkinton, MA, USA). The data are presented as the mean luminescence $\pm \mathrm{SEM}$. 


\section{Immunofluorescence staining}

A549 cells were treated with $10 \mu \mathrm{M}$ MG132 for $6 \mathrm{~h}$. After fixation using $4 \%$ formaldehyde at room temperature for $15 \mathrm{~min}$, the cells were incubated overnight at $4^{\circ} \mathrm{C}$ with goat polyclonal anti-SNAIL (1:200; Abcam, MA, USA) and rabbit polyclonal anti-Jab1 (COPS5) protein (1:200, Abcam). The cells were then stained with Alexa Fluor 555-anti-goat IgG (1:500, Thermo Fisher Scientific) and Alexa Fluor 488-anti-rabbit IgG (1:500, Thermo Fisher Scientific) at room temperature for $90 \mathrm{~min}$ and mounted using VECTASHIELD mounting media with DAPI (Vector Laboratories, Burlingame, CA).

\section{Immunohistochemical staining}

A lung cancer tissue microarray, which consists of 353 cores, was obtained from Toyama University Hospital (Toyama, Japan). Detailed clinical and pathologic information, including patient demographics, smoking history, and overall survival, was available for most patients in the lung cancer tissue microarray. This study was approved by the Institutional Review Boards of University of Toyama. Immunohistochemical staining was performed using BOND-III Fully Automated IHC (Leica Biosystems) at Pathology Institute Corporation (Toyama, Japan). Sections were cut and deparaffinized through graded alcohol and xylene. After antigen retrieval using BOND ER1 buffer, the sections were incubated for 30 minutes at room temperature with goat polyclonal anti-SNAIL (diluted at 1:200; Abcam, MA, USA) and rabbit polyclonal anti-Jab1 (COPS5) protein (1:200, Abcam). After incubation with rabbit polyclonal antigoat serum (1:1000, Bethyl Laboratories, Montgomery, TX, USA), the sections were incubated for 30 minutes at room temperature with BOND Polymer Refine Detection System-HRP (Leica). Then, endogeneous peroxidase was blocked with 3\% hydrogen peroxidase for 5 minutes, visualized with $\mathrm{DAB}$, and counterstained with hematoxylin, dehydrated, cleared, and mounted with resinous mounting medium. Nuclear and/or cytoplasmic staining for SNAIL or COPS5 was considered to indicate positivity. Image capture and evaluation was performed in a masked manner. We scored immunohistochemical staining as described previously [43, 44]. Briefly, the distribution score (DS), which reflects the distribution of the positive signal among tumor cells, was scored as $0(0 \%), 1(1 \%-50 \%)$, or $2(51 \%-100 \%)$ to reflect the percentage of positive tumor cells among whole tumor cells seen in the same tissue disk. The intensity score (IS), intensity of the signal, was scored as 0 (no signal), 1 (weak), 2 (moderate), or 3 (marked). The sum of DS and IS was converted into negative (DS + IS; 0 or 2) and positive (DS + IS; 3-5) [43, 45]. Among 200 cores containing lung adenocarcinoma tissues, 168 cores were scored by two independent researchers. Finally, 160 cores were classified into same groups by two researchers and used as available cores.

\section{Statistical analysis}

Statistical significance was calculated using Graphpad Prism software (GraphPad Software, Inc, San Diego, CA). More than three means were composed using one-way ANOVA with the Bonferroni correction, and two means were composed using unpaired Student's $t$-test. The correlation between COPS5 and SNAIL expression in immunohistochemical stainings was evaluated by Chisquare test among the patient subgroups. $P<0.05$ was considered statistically significant.

\section{Author contributions}

Conceived and designed the experiments: SY. Performed the experiments: KW SY NK TH YI SK. Wrote the paper: KW SY YH HS JF IS.

\section{ACKNOWLEDGMENTS}

We thank Dr. Seiji Yano (Division of Medical Oncology, Cancer Research Institute, Kanazawa University, Ishikawa, Japan) for providing lung adenocarcinoma cells. We thank all members of the Saiki laboratory for discussions and suggestions.

\section{CONFLICTS OF INTEREST}

The authors declare no conflicts of interest.

\section{FUNDING}

This work was supported in part by by Grant-in-Aid 16K18413 for Young Scientists (B) (S.Y.), Grant-in-Aid 26430158 for Scientific Research (C) (Y.H.), and Grantin-Aid 15K08902 for Scientific Research (C) (I.S.) from the Ministry of Education, Culture, Sports, Science, and Technology, Japan, and Takeda Science Foundation (S.Y.).

\section{REFERENCES}

1. Valastyan S, Weinberg RA. Tumor metastasis: molecular insights and evolving paradigms. Cell. 2011; 147:275-92.

2. Fidler IJ. Tumor heterogeneity and the biology of cancer invasion and metastasis. Cancer Res. 1978; 38:2651-60.

3. Peinado H, Olmeda D, Cano A. Snail, Zeb and bHLH factors in tumour progression: an alliance against the epithelial phenotype? Nat Rev Cancer. 2007; 7:415-28.

4. Hsu DS, Wang HJ, Tai SK, Chou CH, Hsieh CH, Chiu PH, Chen NJ, Yang MH. Acetylation of snail modulates the cytokinome of cancer cells to enhance the recruitment of macrophages. Cancer Cell. 2014; 26:534-48. 
5. Mani SA, Guo W, Liao MJ, Eaton EN, Ayyanan A, Zhou AY, Brooks M, Reinhard F, Zhang CC, Shipitsin M, Campbell LL, Polyak K, Brisken C, et al. The epithelialmesenchymal transition generates cells with properties of stem cells. Cell. 2008; 133:704-15.

6. Kudo-Saito C, Shirako H, Takeuchi T, Kawakami Y. Cancer metastasis is accelerated through immunosuppression during Snail-induced EMT of cancer cells. Cancer Cell. 2009; 15:195-206.

7. Zheng X, Carstens JL, Kim J, Scheible M, Kaye J, Sugimoto H, Wu CC, LeBleu VS, Kalluri R. Epithelial-tomesenchymal transition is dispensable for metastasis but induces chemoresistance in pancreatic cancer. Nature. 2015; 527:525-30.

8. Dong C, Yuan T, Wu Y, Wang Y, Fan TW, Miriyala S, Lin Y, Yao J, Shi J, Kang T, Lorkiewicz P, St Clair D, Hung MC, et al. Loss of FBP1 by Snail-mediated repression provides metabolic advantages in basal-like breast cancer. Cancer Cell. 2013; 23:316-31.

9. Wang Y, Shi J, Chai K, Ying X, Zhou BP. The Role of Snail in EMT and Tumorigenesis. Curr Cancer Drug Targets. 2013; 13:963-72.

10. Zheng H, Shen M, Zha YL, Li W, Wei Y, Blanco MA, Ren G, Zhou T, Storz P, Wang HY, Kang Y. PKD1 phosphorylation-dependent degradation of SNAIL by SCFFBXO11 regulates epithelial-mesenchymal transition and metastasis. Cancer Cell. 2014; 26:358-73.

11. Zhou BP, Deng J, Xia W, Xu J, Li YM, Gunduz M, Hung MC. Dual regulation of Snail by GSK-3beta-mediated phosphorylation in control of epithelial-mesenchymal transition. Nat Cell Biol. 2004; 6:931-40.

12. Wu W, Ding H, Cao J, Zhang W. FBXL5 inhibits metastasis of gastric cancer through suppressing Snaill. Cell Physiol Biochem. 2015; 35:1764-72.

13. Vinas-Castells R, Beltran M, Valls G, Gomez I, Garcia JM, Montserrat-Sentis B, Baulida J, Bonilla F, de Herreros AG, Diaz VM. The hypoxia-controlled FBXL14 ubiquitin ligase targets SNAIL1 for proteasome degradation. J Biol Chem. 2010; 285:3794-805.

14. Lander R, Nordin K, LaBonne C. The F-box protein Ppa is a common regulator of core EMT factors Twist, Snail, Slug, and Sip1. J Cell Biol. 2011; 194:17-25.

15. Liu T, Yu J, Deng M, Yin Y, Zhang H, Luo K, Qin B, Li Y, Wu C, Ren T, Han Y, Yin P, Kim J, et al. CDK4/6-dependent activation of DUB3 regulates cancer metastasis through SNAIL1. Nat Commun. 2017; 8:13923.

16. Nijman SM, Luna-Vargas MP, Velds A, Brummelkamp TR, Dirac AM, Sixma TK, Bernards R. A genomic and functional inventory of deubiquitinating enzymes. Cell. 2005; 123:773-86.

17. Kato S, Hayakawa Y, Sakurai H, Saiki I, Yokoyama S. Mesenchymal-transitioned cancer cells instigate the invasion of epithelial cancer cells through secretion of WNT3 and WNT5B. Cancer Sci. 2014; 105:281-9.
18. Thiery JP, Acloque H, Huang RY, Nieto MA. Epithelialmesenchymal transitions in development and disease. Cell. 2009; 139:871-90.

19. Rozen S, Fuzesi-Levi MG, Ben-Nissan G, Mizrachi L, Gabashvili A, Levin Y, Ben-Dor S, Eisenstein M, Sharon M. CSNAP Is a Stoichiometric Subunit of the COP9 Signalosome. Cell Rep. 2015; 13:585-98.

20. Richardson KS, Zundel W. The emerging role of the COP9 signalosome in cancer. Mol Cancer Res. 2005; 3:645-53.

21. Lu R, Hu X, Zhou J, Sun J, Zhu AZ, Xu X, Zheng H, Gao X, Wang X, Jin H, Zhu P, Guo L. COPS5 amplification and overexpression confers tamoxifen-resistance in ERalpha-positive breast cancer by degradation of NCoR. Nat Commun. 2016; 7:12044.

22. Mori M, Yoneda-Kato N, Yoshida A, Kato JY. Stable form of JAB1 enhances proliferation and maintenance of hematopoietic progenitors. J Biol Chem. 2008; 283:29011-21.

23. Kouvaraki MA, Rassidakis GZ, Tian L, Kumar R, Kittas C, Claret FX. Jun activation domain-binding protein 1 expression in breast cancer inversely correlates with the cell cycle inhibitor p27(Kip1). Cancer Res. 2003; 63:2977-81.

24. Pan Y, Zhang Q, Atsaves V, Yang H, Claret FX. Suppression of Jab1/CSN5 induces radio- and chemo-sensitivity in nasopharyngeal carcinoma through changes to the DNA damage and repair pathways. Oncogene. 2013; 32:2756-66.

25. Lee MH, Zhao R, Phan L, Yeung SC. Roles of COP9 signalosome in cancer. Cell Cycle. 2011; 10:3057-66.

26. Zhong G, Li H, Shan T, Zhang N. CSN5 silencing inhibits invasion and arrests cell cycle progression in human colorectal cancer SW480 and LS174T cells in vitro. Int J Clin Exp Pathol. 2015; 8:2809-15.

27. Kim BC, Lee HJ, Park SH, Lee SR, Karpova TS, McNally JG, Felici A, Lee DK, Kim SJ. Jab1/CSN5, a component of the $\mathrm{COP} 9$ signalosome, regulates transforming growth factor beta signaling by binding to Smad7 and promoting its degradation. Mol Cell Biol. 2004; 24:2251-62.

28. Chamovitz DA, Segal D. JAB1/CSN5 and the COP9 signalosome. A complex situation. EMBO Rep. 2001; 2:96-101.

29. Wu Y, Deng J, Rychahou PG, Qiu S, Evers BM, Zhou BP. Stabilization of snail by NF-kappaB is required for inflammation-induced cell migration and invasion. Cancer Cell. 2009; 15:416-28.

30. Fang L, Lu W, Choi HH, Yeung SC, Tung JY, Hsiao CD, Fuentes-Mattei E, Menter D, Chen C, Wang L, Wang J, Lee MH. ERK2-Dependent Phosphorylation of CSN6 Is Critical in Colorectal Cancer Development. Cancer Cell. 2015; 28:183-97.

31. Vinas-Castells R, Frias A, Robles-Lanuza E, Zhang K, Longmore GD, Garcia de Herreros A, Diaz VM. Nuclear ubiquitination by FBXL5 modulates Snail1 DNA binding and stability. Nucleic Acids Res. 2014; 42:1079-94.

32. Zhou Y, Wu J, Fu X, Du W, Zhou L, Meng X, Yu H, Lin J, Ye W, Liu J, Peng H, Liu RY, Pan C, et al. OTUB1 promotes 
metastasis and serves as a marker of poor prognosis in colorectal cancer. Mol Cancer. 2014; 13:258.

33. Tomida S, Takeuchi T, Shimada Y, Arima C, Matsuo K, Mitsudomi T, Yatabe Y, Takahashi T. Relapse-related molecular signature in lung adenocarcinomas identifies patients with dismal prognosis. J Clin Oncol. 2009; 27:2793-9.

34. Yamauchi M, Yamaguchi R, Nakata A, Kohno T, Nagasaki M, Shimamura T, Imoto S, Saito A, Ueno K, Hatanaka Y, Yoshida R, Higuchi T, Nomura M, et al. Epidermal growth factor receptor tyrosine kinase defines critical prognostic genes of stage I lung adenocarcinoma. PLoS One. 2012; 7:e43923.

35. Mizuno H, Kitada K, Nakai K, Sarai A. PrognoScan: a new database for meta-analysis of the prognostic value of genes. BMC Med Genomics. 2009; 2:18.

36. Hou J, Liu G, Yuan Y, Wang D, Jiao P, Xing L, Pan Y. Increased Jab1/COPS5 is associated with therapeutic response and adverse outcome in lung cancer and breast cancer patients. Oncotarget. 2017; 8:97504-15. https://doi. org/10.18632/oncotarget.22146.

37. Dong Y, Sui L, Watanabe Y, Yamaguchi F, Hatano N, Tokuda M. Prognostic significance of Jab1 expression in laryngeal squamous cell carcinomas. Clin Cancer Res. 2005; 11:259-66.

38. Adler AS, Littlepage LE, Lin M, Kawahara TL, Wong DJ, Werb Z, Chang HY. CSN5 isopeptidase activity links COP9 signalosome activation to breast cancer progression. Cancer Res. 2008; 68:506-15.

39. Kouvaraki MA, Korapati AL, Rassidakis GZ, Tian L, Zhang Q, Chiao P, Ho L, Evans DB, Claret FX. Potential role of Jun activation domain-binding protein 1 as a negative regulator of p27kip1 in pancreatic adenocarcinoma. Cancer Res. 2006; 66:8581-9.

40. Hsu MC, Huang CC, Chang HC, Hu TH, Hung WC. Overexpression of Jab1 in hepatocellular carcinoma and its inhibition by peroxisome proliferator-activated receptor \{gamma\} ligands in vitro and in vivo. Clin Cancer Res. 2008; 14:4045-52.

41. Kaneto N, Yokoyama S, Hayakawa Y, Kato S, Sakurai H, Saiki I. RAC1 inhibition as a therapeutic target for gefitinibresistant non-small-cell lung cancer. Cancer Sci. 2014; 105:788-94.

42. Kubota SI, Takahashi K, Nishida J, Morishita Y, Ehata S, Tainaka K, Miyazono K, Ueda HR. Whole-Body Profiling of Cancer Metastasis with Single-Cell Resolution. Cell Rep. 2017; 20:236-50.

43. Fukuoka J, Fujii T, Shih JH, Dracheva T, Meerzaman D, Player A, Hong K, Settnek S, Gupta A, Buetow K, Hewitt S, Travis WD, Jen J. Chromatin remodeling factors and BRM/ BRG1 expression as prognostic indicators in non-small cell lung cancer. Clin Cancer Res. 2004; 10:4314-24.

44. Tsurutani J, Fukuoka J, Tsurutani H, Shih JH, Hewitt SM, Travis WD, Jen J, Dennis PA. Evaluation of two phosphorylation sites improves the prognostic significance of Akt activation in non-small-cell lung cancer tumors. J Clin Oncol. 2006; 24:306-14.

45. He P, Varticovski L, Bowman ED, Fukuoka J, Welsh JA, Miura K, Jen J, Gabrielson E, Brambilla E, Travis WD, Harris CC. Identification of carboxypeptidase E and gamma-glutamyl hydrolase as biomarkers for pulmonary neuroendocrine tumors by cDNA microarray. Hum Pathol. 2004; 35:1196-209. 